\title{
EFEITO DA MODIFICAÇÃO QUÍMICA POR REAÇÃO DE CROSS-LINKING NAS PROPRIEDADES DO AMIDO DE SEMENTE DE ABACATE (Persea americana Mill)
}

\author{
IZIS RAFAELA ALVES SILVA * \\ FABÍOLA SAMARA MEDEIROS DE ALBUQUERQUE ** \\ JAILANE DE SOUZA AQUINO *** \\ VICENTE QUEIROGA NETO ****
}

\begin{abstract}
O objetivo deste trabalho foi obter informações a respeito das propriedades do amido da semente de abacate cv. "Manteiga" e submetê-lo à modificação química por cross-linking, visando obter características reológicas aplicáveis à elaboração de produtos alimentícios. O amido nativo extraído das sementes apresentou teor de amido de $74,47 \%$ e rendimento para o processo de extração utilizado de 11,36 \%. O grau de substituição (GS) do amido nativo por cross-linking foi de 0,006 . Os grânulos de amido nativo e modificado apresentaram formato oval e circular, cujas dimensões variaram de 26 a 26,34 $\mu \mathrm{m}$ para o diâmetro menor e de 35,75 a 36,85 $\mu \mathrm{m}$ para o diâmetro maior. Verificou-se padrão de cristalinidade do tipo $\mathrm{C}$, mostrado por difratometria de raios $\mathrm{X}$. O amido modificado por cross-linking apresentava melhores caracteristicas reológicas que os grânulos do amido nativo, podendo ser aplicado em alimentos para bebês, semi-prontos para serem levados ao forno de microondas, produtos esterilizados, doces e alimentos congelados.
\end{abstract} ABACATE.

* Mestre em Ciência e Tecnologia de Alimentos, Docente, Departamento de Tecnologia em Alimentos, Instituto Federal de Educação, Ciência e Tecnologia Sertão Pernambucano, IF Sertão, Petrolina, PE (e-mail: izis.rafaela@gmail.com).

** Mestre em Ciência e Tecnologia de Alimentos, Departamento de Engenharia de Alimentos, Universidade Federal da Paraíba (UFPB), João Pessoa, PB (e-mail: fabiola-samara2011@hotmail.com).

*** Doutora em Nutrição, Docente, Departamento de Nutrição, UFPB, João Pessoa, PB (e-mail: lalaaquino@ hotmail.com).

**** Doutor em Ciência e Tecnologia de Alimentos, Docente, Unidade Acadêmica de Ciências Biológicas, Universidade Federal de Campina Grande (UFCG), Patos, PB (e-mail: vqneto@cstr.ufcg.edu.br). 


\section{INTRODUÇÃO}

O abacate (Persea americana Mill), fonte de vitaminas, fibras e lipídeos pode ser utilizado tanto na forma "in natura" como manufaturado. A polpa de abacate, por apresentar elevado teor de lipídeo, pode representar opção para a indústria de óleo dependendo do cultivar, porém a sua extração gera biomassa formada por casca e semente (SALGADO et al., 2008). Tango, Carvalho e Soares (2004), avaliando diversos tipos de abacate encontraram variação no percentual de amido das sementes entre 7,8 e 29,3 \%, sendo a média geral de 20,1\%. Assim, a semente de abacate pode ser usada como nova fonte de amido, além de representar solução para o aproveitamento de biomassas geradas por indústrias de processamento da fruta.

O amido, polímero natural, é composto basicamente pelos polissacarídeos amilose e amilopectina. Trata-se do principal carboidrato de reserva das plantas que constitui fonte de energia essencial para muitos organismos, especialmente humanos. O amido assume grande importância para a tecnologia de alimentos devido às propriedades funcionais que apresenta, como poder de inchamento e solubilidade, capacidade de absorção de água e óleo e capacidade de formar gel, entre outros (MIRMOGHTADAIE, KADIVAR e SHAHEDI, 2009).

Apesar de suas características notáveis, o amido nativo apresenta limitações como, faixa estreita de viscosidade, retrogradação em baixa temperatura e textura indesejável, sendo necessária a busca de alternativas para suprir essas limitações. Uma delas seria a realização de modificações no amido nativo a fim de atender a demanda de propriedades peculiares dos produtos alimentícios. Dessa forma, amidos de diferentes fontes são submetidos a modificações pelas vias química, física, enzimática ou combinação de diferentes vias, tendo suas propriedades nativas tecnologicamente melhoradas (MBOUGUENG et al., 2011).

A modificação química por reação de cross-linking resulta da ação de reagentes bi ou polifuncionais, capazes de reagir com mais de um grupo hidroxila, formando ligações cruzadas que proporcionam o controle da textura, tolerância ao aquecimento, à acidez e à agitação mecânica (CARMONA-GARCIA et al., 2009).

Este trabalho teve como objetivo agregar valor à semente de abacate cv. "Manteiga", como fonte amilácea, mediante modificação química de seu amido nativo por cross-linking e analisar a eficiência da modificação por meio de suas características reológicas.

\section{MATERIAL E MÉTODOS}

\subsection{MATERIAL EXPERIMENTAL}

Para a realização do experimento foram utilizados $10 \mathrm{~kg}$ de abacate (Persea americana Mill) cv. "Manteiga", adquiridos no Mercado Produtor do município de Juazeiro (BA). Os frutos foram selecionados de acordo com o estádio de maturação e similaridade na cor das cascas.

\subsection{MÉTODOS}

\subsubsection{Extração do amido}

Obteve-se o extrato amiláceo da semente de abacate cv. "Manteiga" de acordo com adaptações do método descrito por Loos, Hood e Grahan (1981). As sementes, depois de lavadas e cortadas em pequenos pedaços, foram colocadas em infusão com água destilada contendo metabissulfito de sódio $\left(0,2 \mathrm{~g} .100 \mathrm{~g}^{-1}\right)$ durante $48 \mathrm{~h}$ em refrigeração. $\mathrm{O}$ amido foi extraído pela trituração da matéria-prima com solução de metabissulfito de sódio $0,2 \mathrm{~g} .100 \mathrm{~g}^{-1} \mathrm{em}$ liquidificador por 30 min. Após a homogeneização, peneirou-se a mistura em malha de 200 mesh $(0,074 \mathrm{~mm})$. Em seguida, foram realizadas duas decantações por $24 \mathrm{~h}$ com resuspensão em solução de metabissulfito 
de sódio 0,2 g.100 g-1 e centrifugação a $5000 \mathrm{~g}$ por $15 \mathrm{mim}$, sendo o sobrenadante descartado. Retirou-se a mucilagem formada na superfície do resíduo com auxílio de espátula. $O$ amido foi liofilizado (Terroni Equipamentos LTDA, modelo LS 3000, São Paulo-SP, Brasil), à temperatura de $-45{ }^{\circ} \mathrm{C}$ e armazenado em recipiente seco e limpo sob refrigeração. Calculou-se o rendimento do amido da semente de abacate cv. "Manteiga" dividindo a massa do amido seco após extração pela massa da semente sem casca vezes 100 .

\subsubsection{Composição centesimal do amido}

Determinou-se a composição centesimal do amido nativo (amido, umidade, proteínas, lipídeos e cinzas) de acordo com os métodos do Instituto Adolfo Lutz (2005).

\subsubsection{Modificação do amido}

\subsubsection{Cross-linking e determinação do grau de substituição}

A modificação do amido por cross-linking foi realizada de acordo com o método descrito por Woo (1999). Uma massa de $100 \mathrm{~g}$ de amido, $10 \mathrm{~g}$ de sulfato de sódio e $12 \mathrm{~g}$ da mistura (99:1) de trimetafosfato de sódio e tripolifosfato de sódio, respectivamente, foram misturados em água ( $140 \mathrm{~mL}$ ). A mistura foi ajustada para $\mathrm{pH} 11 \mathrm{com} \mathrm{NaOH} 1 \mathrm{M}$ e submetida à agitação por $3 \mathrm{~h}$ a $45^{\circ} \mathrm{C}$. Após esse período, ajustou-se o $\mathrm{pH}$ da dispersão para $6,5 \mathrm{com} \mathrm{HCl} 1 \mathrm{M}$. A dispersão foi centrifugada a $3.000 \mathrm{~g}$ por $10 \mathrm{~min}$, lavada com água destilada (4 vezes), seca em estufa de circulação forçada a $40 \pm 2{ }^{\circ} \mathrm{C}$ durante 1 noite. $\mathrm{O}$ amido modificado foi pulverizado, peneirado a 200 mesh $(0,074 \mathrm{~mm}) \mathrm{e}$ armazenado sob refrigeração em embalagem fechada.

Quantificou-se o teor de fósforo das amidos antes e após o cross-linking de acordo com o método descrito por Ranganna (1991). O percentual de fósforo incorporado foi usado na equação de Smith e Caruso (1964) para obtenção do grau de substituição (GS):

$$
G S=\frac{162 P}{3100-102 P}
$$

Em que: $P$ = percentual de fósforo incorporado na amostra de amido, 3100 e 102 são constantes.

\subsubsection{Estrutura dos grânulos}

\subsubsection{Microscopia eletrônica de varredura (MEV)}

Preparou-se uma dispersão de aproximadamente $2 \mathrm{~g} .100 \mathrm{~mL}^{-1}$ do amido nativo e modificado em álcool isopropílico, sendo colocadas algumas gotas dessa dispersão sobre fita adesiva colada à base de suporte metálico. Após a evaporação do solvente, o material seco foi recoberto com ouro (sputtering) e a amostra analisada em microscópio eletrônico de varredura digital, modelo LEO-1430 (USLU e POLAT, 2012).

\subsubsection{Microscopia óptica (MO)}

Três lâminas para cada amostra foram preparadas com duas gotas de suspensão de $100 \mathrm{mg}$ de amido em $5 \mathrm{~mL}$ de solução 1:1 de glicerina:água e cobertas por lamínula. De cada lâmina foram tomadas medidas de 100 grânulos (YONEMOTO, CALORI-DOMINGUES, FRANCO, 2007). 


\subsubsection{Difração de raios X (DRX)}

O difratograma de raios $\mathrm{X}$ foi obtido a partir de amido na forma de pó, contendo cerca de $11 \%$ de umidade. $O$ intervalo dos ângulos $2 \theta$ variou de 4 a $60^{\circ}$ em Difratômetro D 5000 (Marca Siemens), na velocidade de $1,2^{\circ}$ por minuto operando com potência de $40 \mathrm{kV}$ por $20 \mathrm{~mA}$ (ZOBEL, 1964).

\subsubsection{Propriedades funcionais}

O poder de intumescimento e a solubilidade dos amidos foram determinados segundo o método descrito por Leach, Mccowen e Schoch (1959). Usou-se o método de Beuchat (1977) para determinar a capacidade de absorção de água e de óleo dos amidos nativo e modificado. A avaliação da estabilidade do gel de amido em quatro ciclos de congelamento e descongelamento foi realizada pelo método de White, Abbas e Johnson (1989).

Determinou-se a claridade de pasta conforme Craig et al. (1989), utilizando suspensões de amido $\left(0,3 \mathrm{~g} .100 \mathrm{~mL}^{-1}\right)$. Para monitorar a tendência à retrogradação, as amostras foram armazenadas por 24 horas a $4^{\circ} \mathrm{C}$ e depois resfriadas à temperatura ambiente para a determinação da transmitância no primeiro e no sétimo dias de estocagem.

A viscosidade das amostras foi determinada em Analisador Rápido de Viscosidade (RVA-4), com o programa Thermocline for Windows versão 2.3 (Newport Scientific Pty Ltd.), segundo a metodologia 162 proposta pela International Association for Cereal Science And Techonology (ICC, 1995), utilizando-se o perfil Standard1.

Os parâmetros de gelatinização do amido foram determinados de acordo com Lawal e Adebowale (2005), utilizando Calorímetro Exploratório Diferencial (marca Shimadzu), modelo DSC 60, acoplado a software de computador (Pyris, Perkin Elmer, Belerica, MA, USA), em atmosfera de nitrogênio, com fluxo de $50 \mathrm{~mL}$ por min. Adicionou-se o volume de $5 \mu \mathrm{L}$ de água, usando micro pipeta, em $2 \mathrm{mg}$ de cada amostra de amido em cadinhos de alumínio de DSC. Os cadinhos foram hermeticamente fechados, novamente pesados e deixados em repouso por 24 horas em temperatura ambiente para distribuição uniforme da água no amido. Usou-se a faixa de temperatura de escaneamento de 30 a $150^{\circ} \mathrm{C}$ e razão de aquecimento de $10^{\circ} \mathrm{C}$ por minuto. Em todas as medições, o termograma foi registrado com cadinho de DSC vazio e selado como referência.

\subsubsection{Análises estatísticas}

A análise estatística para comparação dos resultados expressos em médias \pm erro padrão foi realizada mediante ANOVA e teste de Tukey ao nível de $5 \%$. Todas as análises estatísticas foram realizadas utilizando-se o programa Assistat versão 7.6 (SILVA e AZEVEDO, 2008).

\section{RESULTADOS E DISCUSSÃO}

\subsection{MODIFICAÇÃO DO AMIDO}

\subsubsection{Composição centesimal e rendimento do processo de extração}

Os resultados da análise de composição centesimal do amido nativo da semente de abacate cv. "Manteiga", juntamente com o rendimento do seu processo de extração encontram-se na Tabela 1. 
TABELA 1 - COMPOSIÇÃO CENTESIMAL DO AMIDO NATIVO E RENDIMENTO DO PROCESSO DE EXTRAÇÃO

\begin{tabular}{cc}
\hline Componentes & $\mathbf{9 . 1 0 0} \mathbf{~ g}^{-1}$ \\
\hline Amido & $74,47 \pm 0,070$ \\
Umidade & $4,57 \pm 0,300$ \\
Proteínas & $0,52 \pm 0,120$ \\
Lipídeos & $0,38 \pm 0,070$ \\
Cinzas & $0,19 \pm 0,005$ \\
Outros Carboidratos & 24,44 \\
\hline Rendimento & 11,36 \\
\hline
\end{tabular}

"Calculado por diferença.

O teor de amido encontrado na semente de abacate $(74,47$ g.100 g-1 $)$ mostrou-se similar ao reportado por Henríquez et al. (2008) para amido de semente de pinhão $\left(77,17 \mathrm{~g}^{100} \mathrm{~g} \mathrm{~g}^{-1}\right)$.

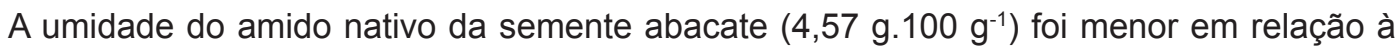
constatada por Rengsutthi e Charoenrein (2011) em amido de semente de jaca (9,59 g.100 g $\left.{ }^{-1}\right)$ e amido de milho $\left(11,74 \mathrm{~g} .100 \mathrm{~g} \mathrm{~g}^{-1}\right)$. Cabe ressaltar que a umidade encontrada no amido nativo foi inferior a $14 \%$, valor que a legislação brasileira (BRASIL, 2005) estabelece como limite para sua boa conservação.

Os baixos percentuais verificados, para proteínas $\left(0,52 \mathrm{~g} .100 \mathrm{~g}^{-1}\right)$, lipídeos $\left(0,38 \mathrm{~g} \cdot 100 \mathrm{~g} \mathrm{~g}^{-1}\right)$ e cinzas $\left(0,19 \mathrm{~g} \cdot 100 \mathrm{~g}^{-1}\right)$ revelam a facilidade com que o amido pode ser extraído e purificado. Henríquez et al. (2008) avaliando amido nativo de semente de pinhão encontraram valores para proteína $\left(0,94 \mathrm{~g} .100 \mathrm{~g}^{-1}\right)$ superiores aos obtidos no presente estudo. Os teores de lipídeos e cinzas verificados por Rengsutthi e Charoenrein (2011) em amido de milho (0,02 g.100 g-1) foram inferiores e as cinzas obtidas de fécula de batata $\left(0,27 \mathrm{~g} .100 \mathrm{~g}^{-1}\right)$ superiores.

A extração do amido da semente de abacate apresentou rendimento de

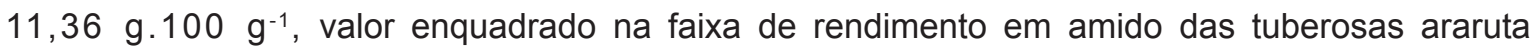
(8-16 g.100 g-1) e mandioquinha salsa $\left(5-23 \mathrm{~g}^{-1} 100 \mathrm{~g} \mathrm{~g}^{-1}\right)$. No entanto, mostrou-se inferior ao rendimento verificado para batata inglesa (18 g.100 g-1), mandioca $\left(22-27 \mathrm{~g}^{-100 ~ \mathrm{~g}^{-1}}\right)$ e inhame (18-23 g.100 g-1) (FRANCO et al., 2002).

\subsubsection{Grau de modificação}

A modificação por cross-linking incorporou quantidade de fósforo equivalente a 0,012 \% ao amido nativo, com consequente grau de substituição de 0,0006 \pm 0 . Os valores encontrados estão de acordo com o que preconiza o Codex Alimentarius (2013) para amido modificado com mistura de trimetafosfato de sódio e tripolifosfato de sódio (limite de $0,4 \%$ de fósforo).

\subsection{ESTRUTURA DOS GRÂNULOS}

\subsubsection{Forma e tamanho}

A microscopia eletrônica de varredura foi utilizada para investigar a morfologia dos grânulos de amido nativo, bem como o efeito da introdução de ligações cruzadas. A modificação química não alterou a forma do grânulo que apresentou formas geométricas similares ao amido nativo, destacando-se pequena quantidade de grânulos circulares e predomínio do formato oval.

$\mathrm{O}$ amido nativo apresentou superficie lisa e sem ranhuras (Figura 1). A modificação química 
pela reação de cross-linking não causou danos significativos à superfície do grânulo, que não revelou bruscas deformações (Figura 2). Esse fato pode estar relacionado ao baixo grau de modificação obtido com o reagente utilizado.

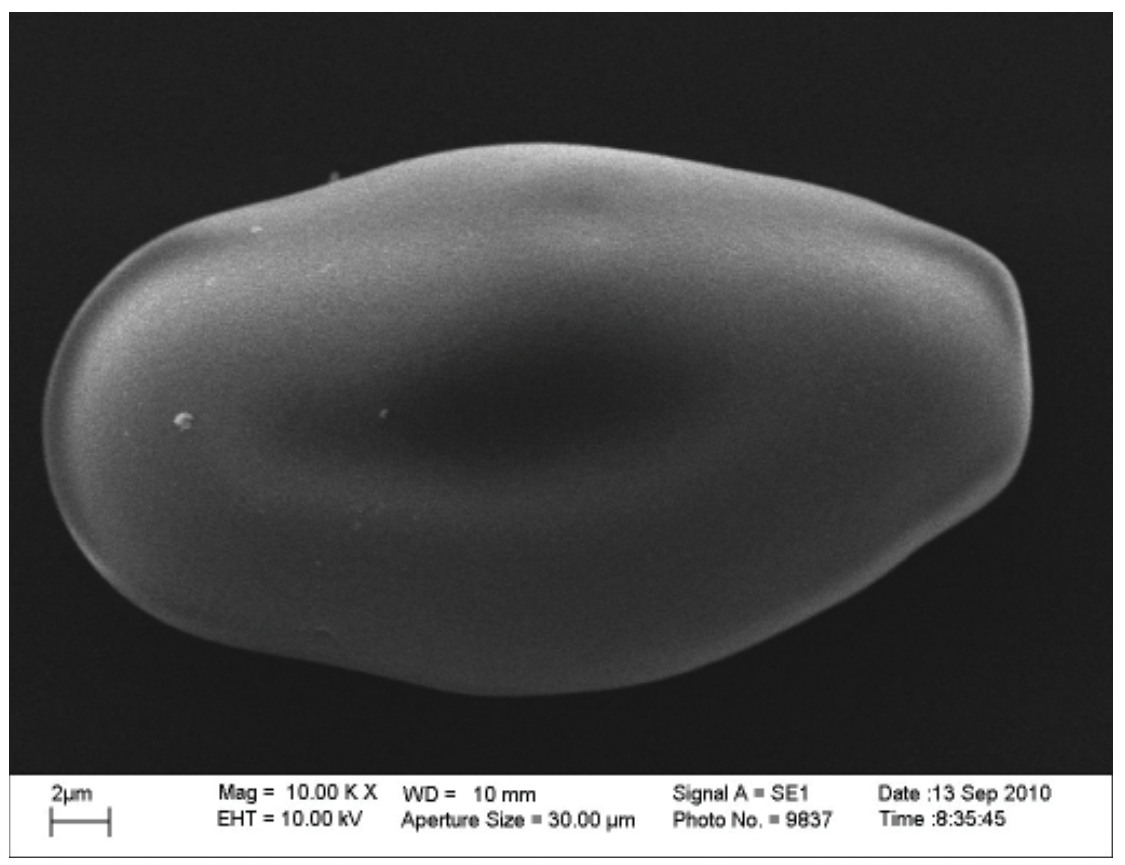

FIGURA 1 - MICROGRAFIA OBTIDA POR MEV (RESOLUÇÃO DE 1000 X) DO AMIDO NATIVO

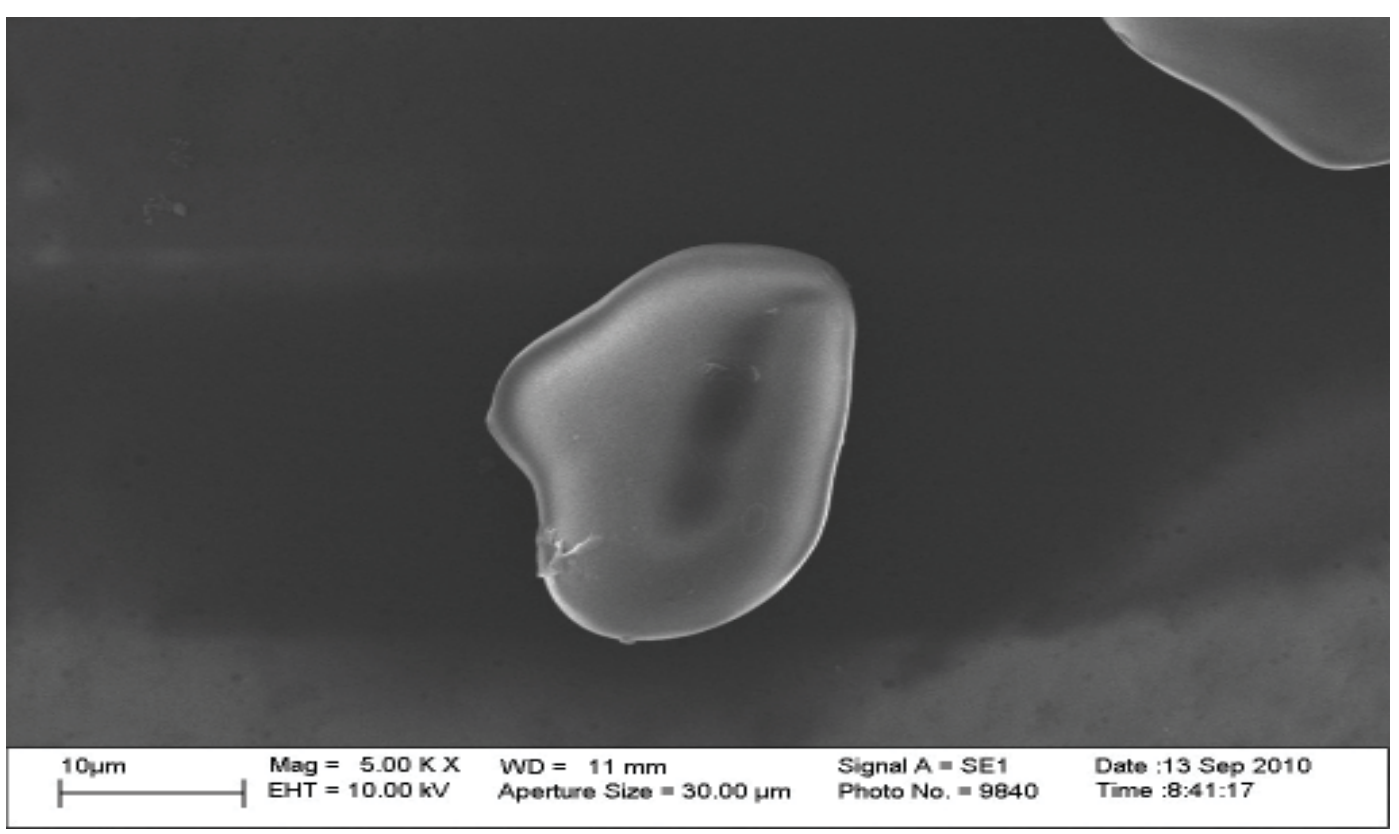

FIGURA 2 - MICROGRAFIA OBTIDA POR MEV (RESOLUÇÃO DE 1000 X) DO AMIDO MODIFICADO POR CROSS-LINKING

As dimensões de diâmetro dos amidos nativo e modificado não mostraram diferenças significativas entre as médias do diâmetro menor e maior de acordo com os resultados obtidos pela análise de microscopia óptica, como ilustra a Figura 3. Comparado a outras fontes botânicas, os amidos nativo e modificado da semente de abacate apresentaram tamanho similar ao diâmetro maior médio da batata $(39,50 \mu \mathrm{m})$ e ao diâmetro menor médio da araruta $(22,05 \mu \mathrm{m})$ (LEONEL, 2007). 


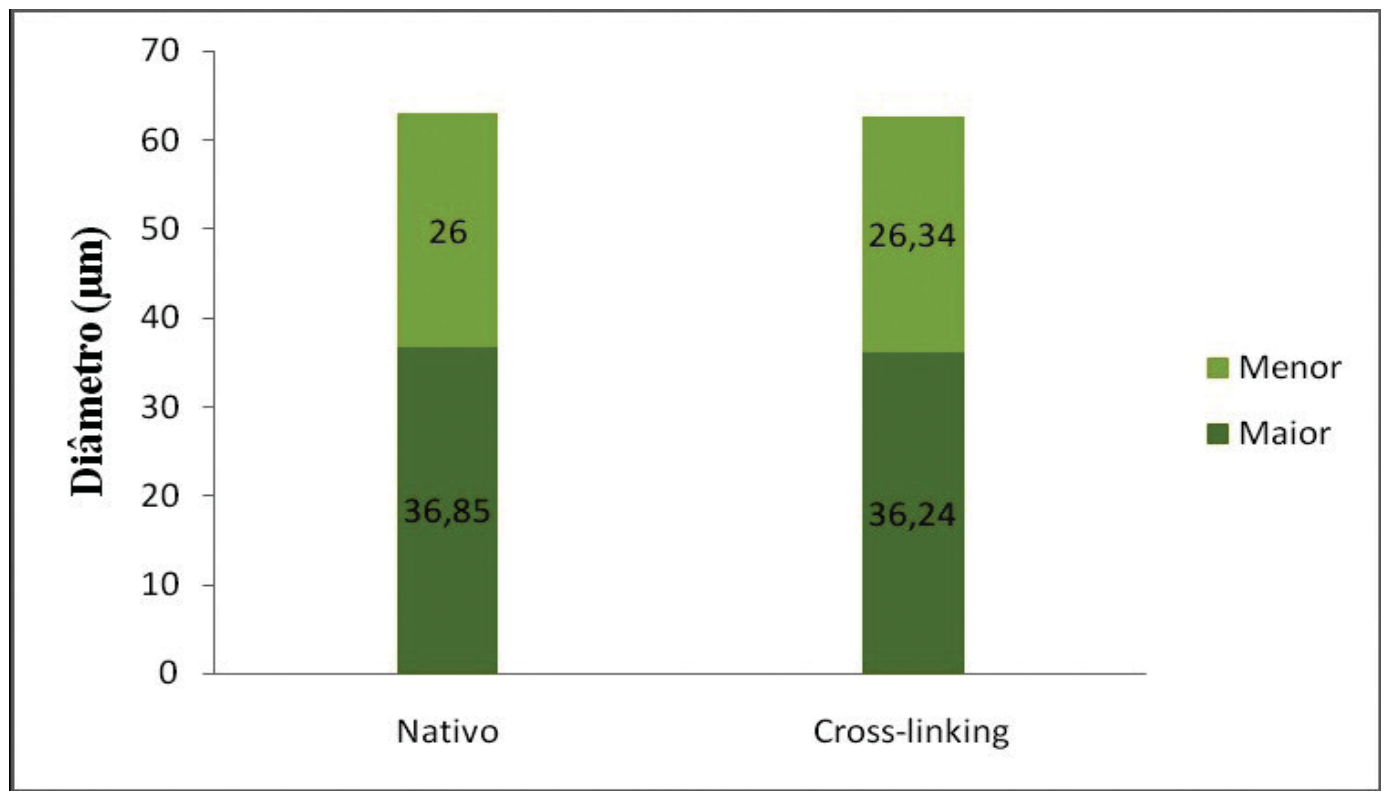

\section{FIGURA 3 - TAMANHO DOS GRÂNULOS DE AMIDOS NATIVO E MODIFICADO DA SEMENTE DE ABACATE}

\subsubsection{Difração de raios X (DRX)}

A difração de raios $X$ foi usada para estabelecer o padrão de cristalinidade e verificar se houve alteração após modificação química do amido nativo. Os difratogramas de raios $\mathrm{X}$ de todas as amostras são mostrados na Figura 4.

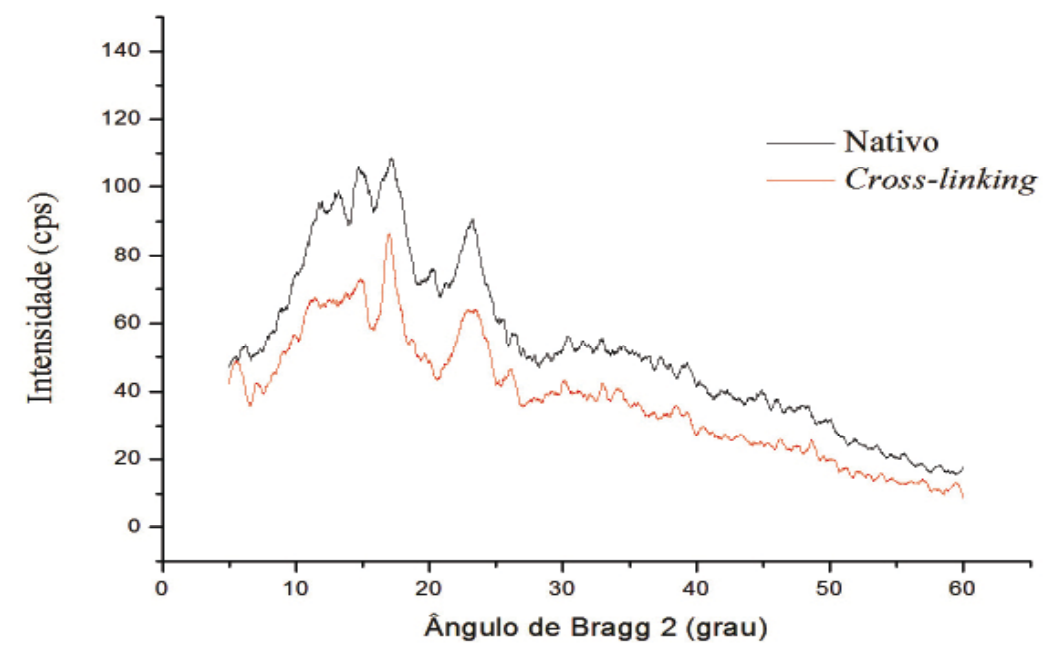

FIGURA 4 - DIFRATOGRAMA DE RAIOS X DOS AMIDOS DA SEMENTE DE ABACATE: NATIVOE MODIFICADO POR CROSS-LINKING

Eixo $X=2 \theta$.

Os grânulos de amido, devido à sua cristalinidade, revelaram na análise de difração em raios $X$ diferentes formas de empacotamento das duplas hélices de amilopectina, chamados cristais dos tipos A, B ou C (CHEETHAM e TAO, 1998). O amido nativo apresentou difratograma de raios $X$ 
característico do tipo $C$ com três picos principais em torno dos ângulos de difração de 14,8; 17,2 e $23,1^{\circ}$ a $2 \mathrm{\theta}$. O tipo $\mathrm{C}$, forma polimórfica, pode ser considerado como a mistura dos tipos $\mathrm{A}$ e $\mathrm{B}$, sendo típico de leguminosas e sementes (VAN SOEST et al., 1996).

$\mathrm{O}$ amido modificado por cross-linking apresentou três picos principais em torno dos ângulos de difração de 14,$8 ; 16,9$ e $23,3^{\circ}$ a $2 \theta$, podendo-se constatar que a modificação química não alterou a estrutura cristalina do grânulo de amido nativo.

\subsection{PROPRIEDADES FUNCIONAIS}

\subsubsection{Poder de intumescimento e solubilidade}

Os dados referentes ao poder de intumescimento e solubilidade dos amidos nativo e modificado por cross-linking da semente de abacate em função da temperatura estão expostos na Tabela 2.

\section{TABELA 2 - EFEITO DA TEMPERATURA SOBRE O PODER DE INTUMESCIMENTO E SOLUBILIDADE DO AMIDO NATIVO E CROSS-LINKING DA SEMENTE DE ABACATE CV. "MANTEIGA"}

\begin{tabular}{|c|c|c|c|c|c|}
\hline \multirow[t]{2}{*}{ Amido } & \multicolumn{5}{|c|}{ Temperaturas } \\
\hline & $55^{\circ} \mathrm{C}$ & $65^{\circ} \mathrm{C}$ & $75^{\circ} \mathrm{C}$ & $85^{\circ} \mathrm{C}$ & $95^{\circ} \mathrm{C}$ \\
\hline \multicolumn{6}{|c|}{ Poder de Intumescimento $\left(\mathbf{g} \cdot \mathbf{g}^{-1}\right)$} \\
\hline Nativo & $0,930^{\mathrm{a}} \pm 0,030$ & $0,970^{\mathrm{a}} \pm 0,020$ & $8,320^{\mathrm{a}} \pm 0,900$ & $10,770^{\mathrm{a}} \pm 0,640$ & $10,940^{\mathrm{a}} \pm 0,190$ \\
\hline Cross-linking & $0,840^{\mathrm{b}} \pm 0,008$ & $0,860^{\mathrm{b}} \pm 0,007$ & $0,860^{\mathrm{b}} \pm 0,010$ & $0,870^{\mathrm{b}} \pm 0,010$ & $0,890^{\mathrm{b}} \pm 0,010$ \\
\hline \multicolumn{6}{|c|}{ Solubilidade $\left(\mathrm{g} .100 \mathrm{~g}^{-1}\right)$} \\
\hline Nativo & $0,012^{\mathrm{b}} \pm 0,001$ & $0,037^{b} \pm 0,006$ & $0,327^{a} \pm 0,021$ & $0,447^{\mathrm{a}} \pm 0,035$ & $0,554^{a} \pm 0,180$ \\
\hline Cross-linking & $0,044^{a} \pm 0,006$ & $0,070^{\mathrm{a}} \pm 0,010$ & $0,074^{b} \pm 0,006$ & $0,080^{b} \pm 0,000$ & $0,090^{b} \pm 0,000$ \\
\hline
\end{tabular}

*Cada valor representa à média e desvio padrão da triplicata

*Diferença significativa entre as colunas representada pelas letras minúsculas.

O poder de intumescimento e a solubilidade aumentaram para todas as amostras de acordo com a elevação da temperatura. $\mathrm{O}$ amido nativo apresentou maior poder de intumescimento em temperaturas superiores a $75^{\circ} \mathrm{C}$, que sugere o início da formação da pasta. No amido modificado por cross-linking, o inchamento se manteve praticamente constante com o aumento da temperatura porque o poder de intumescimento é limitado nesse tipo de modificação devido à presença de fortes ligações entre as moléculas de amido.

O poder de intumescimento geralmente é influenciado pela força de ligação entre as moléculas e pela estrutura molecular da amilopectina. Baixo intumescimento pode ser atribuído à presença de vários cristais formados pela associação de longas cadeias de amilopectina. Maior cristalinidade implica em maior estabilidade granular, o que consequentemente reduz a capacidade de inchamento do grânulo (SINGH et al., 2004).

$\mathrm{O}$ amido nativo apresentou poder de intumescimento de 0,$97 ; 8,32$ e 10,77 g. $\mathrm{g}^{-1}$ nas temperaturas de 65,75 e $85^{\circ} \mathrm{C}$, respectivamente. Muccillo (2009) estudando amido de pinhão nativo (Araucaria brasiliense) obteve 1,91; 3,84 e 11,08 g. g-1 nas temperaturas de 50, 60 e $70^{\circ} \mathrm{C}$.

Os dados revelaram que a modificação por cross-linking reduziu a capacidade de intumescimento do amido nativo. Mirmoghtadaie, Kadivar e Shahedi (2009) estudando amido de aveia nativo e modificado por cross-linking em diferentes concentrações observaram o mesmo comportamento. Carmona-Garcia et al. (2009) obtiveram resultado semelhante utilizando a mesma 
modificação nas temperaturas de 80 e $90^{\circ} \mathrm{C}$ em amido nativo de banana. Os resultados confirmaram que a introdução de grupos de fosfato em amido reforça as ligações de hidrogênio nas moléculas do grânulo, fazendo com que a gelatinização ocorra em temperatura superior.

O inchamento do grânulo ocorre simultaneamente à perda de birrefringência e precede a solubilização. A solubilidade dos amidos apresentou comportamento similar ao poder de intumescimento, como pode ser visto na Tabela 2 (a solubilidade aumentou de acordo com a temperatura).

$\mathrm{O}$ amido nativo apresentou percentual de solubilidade de 0,$012 ; 0,037 ; 0,327 ; 0,447 \mathrm{e}$ $0,554 \%$ nas temperaturas de $55,65,75,85$ e $95^{\circ} \mathrm{C}$, respectivamente. Os valores encontrados no presente estudo foram inferiores aos relatados por Adebowale, Afolabi e Olu-Owolabi (2006) avaliando amido nativo de feijão espada nas temperaturas de $50,60,70,80$ e $90^{\circ} \mathrm{C}$.

Os amidos com ligações cruzadas incham menos e são menos solúveis que amidos não modificados devido, provavelmente, ao intercruzamento. Carmona-Garcia et al. (2009) estudando amido modificado por cross-linking em banana verificaram que a modificação diminuiu a solubilidade do grânulo nas temperaturas de 70,80 e $90^{\circ} \mathrm{C}$ em relação ao amido nativo.

\subsubsection{Viscosidade}

Os resultados dos parâmetros da viscosidade dos amidos nativo e modificado foram agrupados de acordo com os valores médios obtidos na análise RVA (Tabela 3).

\section{TABELA 3 - PARÂMETROS PRINCIPAIS DA VISCOSIDADE DO AMIDO NATIVO E CROSS-LINKING DA SEMENTE DE ABACATE POR MEIO DE CURVAS AVALIADAS PELO ANALISADOR RÁPIDO DE VISCOSIDADE}

\begin{tabular}{lcc}
\hline \multicolumn{1}{c}{ Parâmetros (RVA) } & Nativo & Cross-linking \\
\hline Temperatura da pasta $\left({ }^{\circ} \mathrm{C}\right)$ & 80,22 & 95,20 \\
Viscosidade de pico & 284,34 & 10,08 \\
Viscosidade mínima & 163,34 & 4,25 \\
Quebra (Breakdown) & 121 & 5,83 \\
Viscosidade final & 467,42 & 4,25 \\
Tendência a retrogradação & 304,08 & 0 \\
$($ Setback) & 5 & 6,60 \\
\hline Tempo de pico (min) & & \\
\hline
\end{tabular}

O amido modificado apresentou maior temperatura de pasta e menor viscosidade de pico em relação ao amido nativo, o que pode ser explicado pela formação de ligações cruzadas que dificultam a gelatinização.

O amido modificado por cross-linking mostrou-se estável frente ao aquecimento, apresentando quebra reduzida quando comparado ao nativo (elevado pico de viscosidade). Segundo Singh et al. (2003), os amidos com alto grau de inchamento são menos resistentes à quebra no cozimento exibindo, consequentemente, diminuição significativa na viscosidade depois de atingir o valor máximo. $\mathrm{O}$ amido modificado por cross-linking apresentou menor tendência a retrogradação em relação ao amido nativo. 


\subsubsection{Claridade da pasta e tendência a retrogradação}

O amido nativo apresentou pasta opaca com valor de 2,2 \% de transmitância (Tabela 4) e o modificado por cross-linking pasta moderadamente transparente.

TABELA 4 - EFEITO DO TEMPO DE ESTOCAGEM NA TRANSPARÊNCIA DO GEL DOS AMIDOS DA SEMENTE DE ABACATE NATIVO E MODIFICADO POR CROSS-LINKING

\begin{tabular}{|c|c|c|c|}
\hline \multirow{3}{*}{ Amido } & \multicolumn{3}{|c|}{ \% Transmitância } \\
\hline & \multirow[t]{2}{*}{ Claridade da Pasta } & \multicolumn{2}{|c|}{ Retrogradação } \\
\hline & & $1^{\circ} \mathrm{dia}$ & $7^{\circ}$ dia \\
\hline Nativo & $2,20^{\mathrm{b}} \pm 0,20$ & $0,40^{\mathrm{b}} \pm 0,00$ & $0,20^{\mathrm{b}} \pm 0,00$ \\
\hline Cross-linking & $42,25^{a} \pm 4,15$ & $43,50^{a} \pm 5,51$ & $47,30^{\mathrm{a}} \pm 4,67$ \\
\hline
\end{tabular}

"Cada valor representa a média e desvio padrão da triplicata.

'Diferença significativa entre as colunas representada pelas letras minúsculas.

O estudo sobre o efeito do período de armazenamento na transparência do gel revelou que o amido nativo estocado durante sete dias sob refrigeração evidenciou pequena redução na claridade de sua pasta. Segundo Stahl et al. (2007) isso acontece porque durante a estocagem ocorre a retrogradação que torna as dispersões de amido opacas dando origem a gel rígido.

A pasta obtida do amido modificado foi ficando mais translúcida com o tempo, indicando que as ligações de hidrogênio formadas pelo intercruzamento impedem a reorganização das cadeias de amilose (retrogradação), permitindo maior passagem de luz. Tal fato confirma que o amido modificado por cross-linking é mais resistente à retrogradação que o amido nativo.

\subsubsection{Capacidade de absorção em água e óleo}

Os resultados expostos na Figura 5 mostram que a modificação por cross-linking (70,48 g $\mathrm{H}_{2} \mathrm{O} .100 \mathrm{~g}^{-1}$ amido) reduziu a tendência do amido nativo $\left(79,88 \mathrm{~g} \mathrm{H}_{2} \mathrm{O} .100 \mathrm{~g}^{-1}\right.$ amido) em absorver água. As interações entre a ligação de hidrogênio e as moléculas do amido ao se tornarem mais numerosas e mais fortes reduziram as superfícies disponíveis para tais ligações com as moléculas de água.

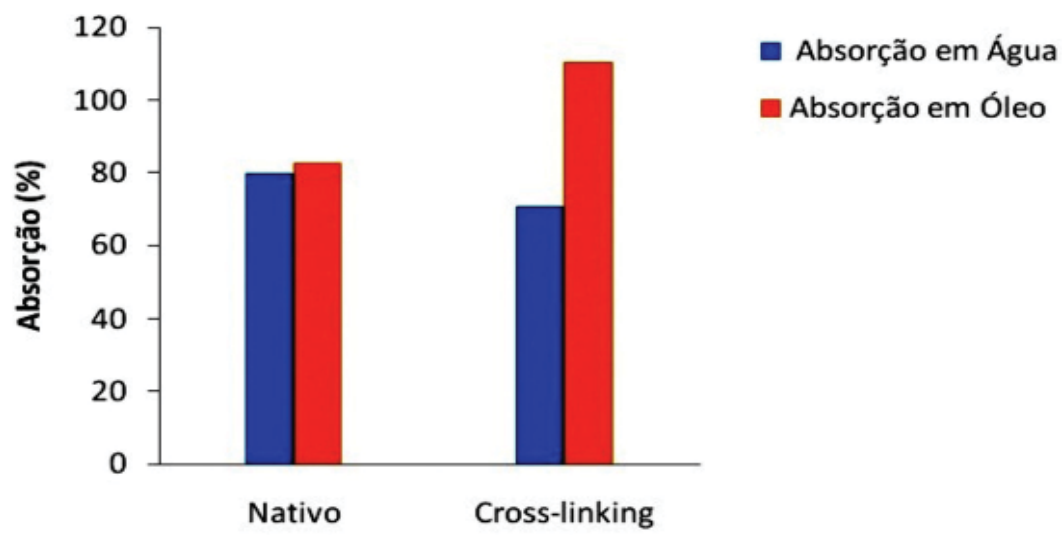

\section{FIGURA 5 - CAPACIDADE DE ABSORÇÃO DE ÁGUA E ÓLEO EM AMIDOS NATIVO E MODIFICADO DA SEMENTE DE ABACATE CV. "MANTEIGA"}

Observou-se também que a tendência hidrofóbica do amido nativo $(82,71 \mathrm{~g}$ óleo. $100 \mathrm{~g}^{-1}$ amido) melhorou após a modificação por cross-linking (110,27 g óleo.100 g-1 amido). Esse comportamento ocorreu porque a introdução de grupos funcionais melhora a afinidade com grupos apolares. 


\subsubsection{Estabilidade ao congelamento e descongelamento}

A análise de resistência a ciclos de congelamento e descongelamento torna-se importante para caracterizar o amido em termos de sua aplicabilidade em alimentos que devem ser refrigerados ou congelados, visto que a liberação de água prejudica a qualidade do produto final.

A liberação de água das pastas de amido quando submetidas a ciclos de congelamento e descongelamento pode ser observada pelos dados da Tabela 5. Os valores foram expressos em porcentagem de água liberada em relação à massa inicial da pasta.

TABELA 5 - SINÉRESE DAS PASTAS DE AMIDOS NATIVO E MODIFICADO DA SEMENTE DE ABACATE EM QUATRO CICLOS DE CONGELAMENTO E DESCONGELAMENTO

\begin{tabular}{lcccc}
\hline Amido & $\mathbf{1}$ ciclo & 2 ciclo & 3 ciclo & 4 ciclo \\
\hline Nativo & $7,05^{\mathrm{b}} \pm 0,87$ & $12,89^{\mathrm{a}} \pm 1,34$ & $2,06^{\mathrm{a}} \pm 0,22$ & $1,12^{\mathrm{a}} \pm 0,85$ \\
Cross-linking & $63,38^{\mathrm{a}} \pm 1,63$ & $1,80^{\mathrm{b}} \pm 0,76$ & $0,00^{\mathrm{b}} \pm 0,00$ & $0,00^{\mathrm{a}} \pm 0,00$ \\
\hline
\end{tabular}

"Cada valor representa a média e desvio padrão da triplicata.

*Diferença significativa entre as colunas representada pelas letras minúsculas.

A percentagem de água liberada pelo amido nativo $(7,05 \%)$ no primeiro ciclo foi inferior aos valores encontrados no amido modificado por cross-linking (63,38 \%). A partir do segundo ciclo, os percentuais de água liberada pelo amido modificado ficaram bem abaixo do encontrado no primeiro ciclo, enquanto o amido nativo continuou a liberar água. No terceiro e quarto ciclos houve redução do percentual de água liberada pelo amido nativo e o amido modificado já não apresentava mais sinérese.

Shirai et al. (2007), estudando os amidos nativos de batata, batata doce, mandioquinha, mandioca, milho irregular e ceroso verificaram baixa estabilidade das amostras frente a ciclos de congelamento e descongelamento em razão do aumento da liberação de água com o passar do tempo.

Deetae et al. (2008) avaliaram a estabilidade de amidos nativo e modificado por crosslinking de arroz frente ao congelamento e descongelamento. Verificaram que a modificação reduziu o percentual de água liberado do primeiro ao quarto ciclo, enquanto no quinto ciclo houve aumento da sinérese. Para Mirmoghtadaie, Kadivar e Shahedi (2009), a modificação por acetilação e crosslinking de amido de aveia em diferentes concentrações apresentou maior liberação de água com o passar dos dias em relação ao amido nativo.

\subsubsection{Análise térmica: gelatinização}

As propriedades térmicas das amostras de amido nativo e modificado são apresentadas na Tabela 6.

\section{TABELA 6 - TEMPERATURAS E ENTALPIA ASSOCIADAS COM A GELATINIZAÇÃO DOS AMIDOS NATIVO E MODIFICADO POR CROSS-LINKING DA SEMENTE DE ABACATE CV. "MANTEIGA"}

\begin{tabular}{lccccc}
\hline \multirow{2}{*}{ Amido } & \multicolumn{5}{c}{ Temperaturas $\left({ }^{\circ} \mathbf{C}\right)$} \\
\cline { 2 - 6 } & $\mathbf{T i}$ & $\mathbf{T p}$ & $\mathbf{T f}$ & $\mathbf{T f}-\mathrm{Ti}$ & $\Delta \mathbf{H}\left(\mathbf{J} . \mathbf{g}^{-1}\right)$ \\
\hline Nativo & 43,17 & 88,57 & 94,65 & 51,48 & 666,34 \\
Cross-linking & 46,10 & 87,37 & 92,60 & 46,50 & 622,71 \\
\hline
\end{tabular}

$\mathrm{Ti}=$ temperatura inicial; $\mathrm{Tp}=$ temperatura de pico; $\mathrm{Tf}=$ temperatura final; $\Delta \mathrm{H}=$ entalpia de gelatinização. 
$\mathrm{O}$ amido modificado mostrou temperatura inicial $\left(46,10^{\circ} \mathrm{C}\right)$ maior do que a encontrada no amido nativo $\left(43,17^{\circ} \mathrm{C}\right)$. Segundo Gunaratne e Corke (2007) isto ocorre porque a modificação por cross-linking reduz o poder de intumescimento, retardando o processo de gelatinização. A amostra modificada por cross-linking apresentou pouco efeito sobre os parâmetros de gelatinização, uma vez que não houve diferença significativa em relação à temperatura de pico endotérmico $\left(87,37^{\circ} \mathrm{C}\right) \mathrm{e}$ temperatura final $\left(92,60^{\circ} \mathrm{C}\right)$. A energia envolvida no processo de gelatinização do amido modificado foi inferior à do amido nativo.

Choi et al. (2009) estudando amido nativo de milho obtiveram valores de 64,$5 ; 70,7 ; 87$, $5^{\circ} \mathrm{C}$ e $7,6 \mathrm{~J} \mathrm{.g}{ }^{-1} \mathrm{C}$ referente a Ti, $\mathrm{Tp}, \mathrm{Tf}$ e $\Delta \mathrm{H}$, respectivamente. Os valores reportados por esses autores divergiram dos valores verificados no presente estudo (Tabela 6), o que pode ser atribuído ao uso de diferentes fontes botânicas. Deetae et al. (2008) estudando amido nativo de arroz constataram valores de 71,$64 ; 76,38 ; 83 ; 50 ; 11 ; 86{ }^{\circ} \mathrm{C}$ e $11,74 \mathrm{J.g}^{-1}$ para Ti, Tp, Tf, Tf-Ti e $\Delta \mathrm{H}$, respectivamente.

Mirmoghtadaie, Kadivar e Shahedi (2009) estudaram amido de aveia modificado por acetilação e cross-linking em níveis variados. Constaram que a temperatura de pico do amido modificado por cross-linking em concentrações de 0,05 \% e 0,1\% apresentaram valores de 62,6 e $63,1{ }^{\circ} \mathrm{C}$, respectivamente, não diferindo do amido nativo $\left(\mathrm{Tp}=62,7^{\circ} \mathrm{C}\right)$. Tal fato mostra-se similar aos resultados encontrados no presente estudo para esse tipo de modificação.

\section{CONCLUSÃO}

A semente de abacate, cv. "Manteiga", apresentou grande potencial como fonte amilácea devido ao seu teor de pureza e rendimento no processo de extração, podendo constituir complementação financeira aos empreendedores de produtos de abacate. Os amidos nativo e modificado apresentaram semelhanças com a araruta e a batata em relação à forma e tamanho dos grânulos. Em termos de cristalinidade, as amostras apresentaram estrutura do tipo C característica de fontes de amido oriundas de sementes. A modificação química por cross-linking melhorou as características reológicas do amido nativo, que pode ser utilizado em alimentos infantis, semiprontos (a serem submetidos ao forno micro-ondas), produtos esterilizados e como agentes ligantes em produtos de confeitaria, sendo ideal para alimentos congelados devido sua baixa tendência à retrogradação.

\section{ABSTRACT}

\section{THE EFFECT OF CHEMICAL MODIFICATION BY CROSS-LINKING REACTION ON THE PROPERTIES OF STARCH MADE OF Persea americana Mill AVOCADO SEEDS}

The aim of this study was to obtain information on the properties of starch from the seed of avocado cv."Butter" and submit it to chemical modification by cross-linking in order to provide rheological characteristics applicable in obtaining food. The nativestarch showed the starch content of $74.47 \%$ and yield on extraction process of $11.36 \%$. The degree of substitution (DS) of native starch by cross-linking was 0.006 . The granules of native and modified starch had oval and circular dimensions, ranging from 26 to $26.34 \mu \mathrm{m}$ for the smaller diameter and 35.75 to $36.85 \mu \mathrm{m}$ for the major diameter. The crystallinity pattern shown by $\mathrm{X}$ ray diffraction was type $\mathrm{C}$. The starch modified by cross-linking showed improvement in the rheological characteristics of native starch granules therefore it can be applied to babyfood, semi-ready food to be taken to the microwave, sterile products, fresh and frozen foods.

KEY-WORDS: THICKENER; CHEMICAL MODIFICATION; CHARACTERISTICS RHEOLOGICAL.

\section{REFERÊNCIAS}

1 ADEBOWALE, K.O.; AFOLABI, T.A.; OLU-OWOLABI, B.I. Functional, physicochemical and retrogradation properties of sword bean (Canavalia gladiata) acetylated and oxidized starches. Carbohydrate Polymers, v. 65, n. 93, p. 101, 2006.

2 BEUCHAT, L.R. Functional and electrophoretic characteristics of succinylated peanut flour protein. Journal of Agricultural and Food Chemistry, v.25, p.258-261, 1977. 
3 BRASIL. Ministério da Saúde. Agência Nacional de Vigilância Sanitária (ANVISA). Resolução RDC n. 263, de 22 de setembro de 2005. Aprova o regulamento técnico para produtos de cereais, amidos, farinhas e farelos. Disponível em: <http//e-legis.anvisa.gov.br/leisref/public/showAct.php?id=18822\&Word=>. Acesso em: 20 jun. 2009.

4 CARMONA-GARCIA, R.; MIRNA, M.S.R.; GUADALUPE, M.M., BELLANIRA; G.M.; LUIS, A.B.P. Effect of the cross-linked reagent type on some morphological, physicochemical and functional characteristics of banana starch (Musa paradisiaca). Carbohydrate Polymers, v. 76, p. n. 117, p. 122, 2009.

5 CHEETHAM, N.W.H.; TAO, L. Solid state NMR studies on the structural and conformational properties of natural maize starches. Carbohydrate Polymers, v. 36, n. 4, p. 277-284, 1998.

$6 \mathrm{CHOI}$, H. S.; KIMB, H.S.; PARK, C.S.; KIMA, B.Y.; BAIK, M.Y. Ultra high pressure (UHP)-assisted acetylation of corn starch. Carbohydrate Polymers, v. 78, p. 862-868, 2009.

7 CRAIG, S.A.S.; MANINGAT, C.C.; SEIB, P.A.; HOSENEY, R.C. Starch paste clarity. Cereal Chemistry, v. 66, n. 3, p. 173$182,1989$.

8 DEETAE, P.; SHOBSNGOB, S.; VARNYANOND, W.; CHINACHOTI, P.; NAIVIKUL, O.; VARAVINIT, S. Preparation, pasting properties and freeze-thaw stability of dual modified crosslink-phosphorylated rice starch. Carbohydrate Polymers, v. 73, p. 351-358, 2008,

9 FRANCO,C.M.L.; DAIUTO, E.R.; DEMIATE, I.M.; CARVALHO, L.J.C.; LEONEL, M.; CEREDA, M.P.; VILPOUX, O.F.; SARMENTO, S.B.S. Série culturas de tuberosas amiláceas latino americanas. São Paulo: Fundação Cargill, 2002.

10 GUNARATNE, A.; CORKE, H. Functional properties of hydroxypropylated cross linked, and hydroxypropylated cross linked tuber and root starches. Cereal Chemistry, v. 84, p. 30-37, 2007.

11 HENRÍQUEZ, C.; WSCOBAR, B.; FIGUEROLA, F.; CHIFFELLE, I.; SPEISKY, H.; ESTÉVEZ, A.M. Characterization of Piñon seed (Araucaria araucana (Mol) K. Koch) and the isolated starch from the seed. Food Chemistry, v. 107, p. 592$601,2008$.

12 INSTITUTO ADOLFO LUTZ. Normas analíticas do Instituto Adolfo Lutz: métodos químicos e físicos para análises de alimentos. 4 ed. Brasília, 2005. 1018 p.

13 INTERNATIONAL ASSOCIATION FOR CEREAL SCIENCE AND TECHNOLOGY. Rapid pasting method using the newport rapid viscoanalyser. Vienna, 1995. 10 p. (Standard, 162).

14 LAWAL, O.S.; ADEBOWALE, K.O. Physicochemical characteristics and thermal properties of chemically modified Jack bean (Canavalia ensiformis) starch. Carbohydrate Polymers, v. 60, p. 331-341, 2005.

15 LEACH, H.W.; MCCOWEN, L.D.; SCHOCH, T.J. Structure of starch granule. I Swelling and solubility patterns of various starches. Cereal Chemistry, v. 36, n.6, p.534-544, 1959.

16 LEONEL, M. Análise da forma e tamanho de grânulos de amidos de diferentes fontes botânicas. Ciênc. Tecnol. Aliment., v. 27, n.3, p.579-588, 2007.

17 LOOS, P.J.; HOOD, L.F.; GRAHAM, H.D. Isolation and characterization of starch from breadfruit. Cereal Chemistry, v. 54, p.282-286, 1981

18 MBOUGUENG, P.D.; TENIN, D.; SCHER, J.; TCHIÉGANG, C. Influence of acetylation on physicochemical, functional and thermal properties of potato and cassava starches. Journal of Food Engineering, v.108, p.320-326, 2012.

19 MIRMOGHTADAIE, L.; KADIVAR, M.; SHAHEDI, M. Effects of cross-linking and acetylation on oat starch properties. Food Chemistry, v. 116, p. 709-713, 2009.

20 MUCCILLO, R.C.S.T. Caracterização e avaliação de amido nativo e modificado de pinhão mediante provas funcionais e térmicas. Tese (Doutorado em Engenharia Química) - Universidade Federal do Rio Grande do Sul, Porto Alegre, 2009.

21 RANGANNA, S. Analysis and quality control for fruit and vegetable products. New Delhi: McGraw-Hill, 1991. 1112 p.

22 RENGSUTTHI, K.; CHAROENREIN, S. Physico-chemical properties of jackfruit seed starch (Artocarpus heterophyllus) and its application as a thickener and stabilizer in chili sauce, Food Science and Technology, v. 44, p. 1309-1313, 2011.

23 SALGADO, J.M.; DANIELI, F.; REGITANO-D'ARCE, M.A.B.; FRIAS, A.; MANSI, D. N. Óleo de abacate (Persea americana Mill) como matéria-prima para a indústria alimentícia. Ciênc. Tecnol. Aliment., Campinas, v.28, p.20-26, 2008.

24 SHIRAI, M.A.; HAAS, A.; FERREIRA, G.F.; MATSUGUMA, L.S.; FRANCO, C. M .L.; DEMIATE, I.M. Características físicoquímicas de amidos modificados por tratamento oxidativo. Ciênc. Tecnol. Aliment., v.27, n.2, p. 239-247, 2007.

25 SILVA, F. de A.S.; AZEVEDO, C.A.V. de. A new version of the Assistant - statistical assistance software. Version 7.5. In: 
WORLD CONGRESS ON COMPUTERS IN AGRICULTURE, 4., 2008. Orlando. Annals...Orlando: American Society of Agricultural Engineers, 2008. p. $393-396$.

26 SINGH, N.; KAUR, M.; SANDHU, K.S.; GURAYA, H.S. Physicochemical, thermal, morphological and pasting properties of starches from some indian black gram (Phaseolus mungo I.) cultivars. Starch/stärke, v.56, n.11, p.535-544, 2004.

27 SINGH, N.; SINGH, J.; KAUR, L.; SODHI, N. S.; GILL, B. S. Morphological, thermal and rheological properties of starches from different botanical sources. Food Chemistry, v. 81, p. 219-231, 2003.

28 SMITH, R.J.; CARUSO, J. Determination of phosphorous. In: BEMILLER, R. J. N.; WHISTLER, M. L.; WOLFROM, L.; SMITH, R. J. (Eds.). Methods in carbohydrate chemistry. New York: Academic Press, 1964. p. 42-44.

29 STAHL, J.A.; LOBATO, L.P.; BOCHI, V.C.; KUBOTA, E.H.; GUTKOSKI, L.C.; EMANUELLI, T. Physicochemical properties of pinhão (Araucária angustifolia) starch phosphates. Food Science and Technology, n.40, p. 1206-1214, 2007.

30 TANGO, J.S.; CARVALHO, C.R.L.; SOARES, N.B. Caracterização física e química de frutos de abacate visando a seu potencial para extração de óleo. Rev. Bras. Frutic., Jaboticabal, v. 26, n. 1, p. 17-23, 2004.

31 USLU,M-K.; POLAT, S. Effects of glyoxal cross-linking on baked starch foam. Carbohydrate Polymers, v.87, p. 19941999, 2012

32 VAN SOEST, J.J.G.; HULLEMAN, S.H.D.; DE WIT, D.; VLIEGENTHART, J.F.G. Crystallinity in starch bioplastics. Industrial Crops and Products, v. 5, p. 11-22, 1996.

33 WHITE, P.J.; ABBAS, I.R.; JOHNSON, L.A. Freeze-thaw stability and refrigerated-storage retrogradation of starches. Starch, v. 41, p. 176-181, 1989.

34 WOO, K.S. Cross-linked, RS4 type resistant starch: preparation and properties. 1999. 181 s. PhD Thesis, University Manhattan, Kansas State, 1999.

35 YONEMOTO, P.G.; CALORI-DOMINGUES, M.A.; FRANCO, C.M.L. Efeito do tamanho dos grânulos nas características estruturais e físico-químicas do amido de trigo. Ciência e Tecnologia de Alimentos, v. 27, n. 4, p. 761-771, 2007.

36 ZOBEL, H. F. X. Ray analysis of starches granules. In: WHISTLER, R.L. (Ed.). Carboydrate chemistry. New York: Academic Press, 1964. p. 109-113. 